\title{
Plataforma educativa para desarrollo de sistemas de software radio mediante modulación QPSK en Octave y Arduino
}

\section{Educational platform for software radio system development through QPSK modulation in Octave and Arduino}

\author{
SILVA-CRUZ, Eric Mario †*, CABALLERO-JULIÁN, Franco Gabriel, PÉREZ-SOLANO, Miguel \\ Ángel y PÉREZ-OJEDA, Cristian
}

Instituto Tecnológico de Oaxaca, México.

ID $1^{\mathrm{er}}$ Autor: Eric Mario, Silva-Cruz / ORC ID: 0000-0002-0496-9682, CVU CONACYT ID: 206891

ID $1^{\text {er }}$ Coautor: Franco Gabriel, Caballero-Julián / ORC ID:0000-0002-5924-7759, CVU CONACYT ID: 88993

ID $2^{\text {do }}$ Coautor: Miguel Ángel, Pérez-Solano / ORC ID: 0000-0001-8300-0935

ID $3^{\text {er }}$ Coautor: Cristian, Pérez-Ojeda / ORC ID: 0000-0003-4414-6231

DOI: $10.35429 /$ JOTE.2020.12.4.22.31

Recibido 20 de Abril, 2020; Aceptado 30 de Junio, 2020

\section{Resumen}

Hoy en día, el desarrollo de sistemas de radio basado en software ha permitido la transmisión de datos a altas velocidades y frecuencias en el orden de Gigahertz (Jovanovic, 2018). Para esto, el sistema digital se caracteriza por emplear técnicas de modulación digital en software mediante símbolos de transmisión en la etapa Digital-End, usando conversores digital-analógico (DAC) $\mathrm{y}$ analógico-digitales (ADC) para variar la frecuencia de muestreo y transmitir los datos en el Front End, (Chien, 2001). Este trabajo presenta una plataforma educativa digital para simular las características de un sistema de Software Radio y no está relacionada a una plataforma web educativa. La presente investigación se enfoca en la caracterización de señales moduladas mediante Octave considerando en primera instancia la modulación en cuadratura QPSK, en donde la transmisión de la señal modulada se realiza mediante la tarjeta Arduino en un puerto digital, con una aproximación digital - analógica (DAC) utilizando modulación PWM, un filtro RC y un circuito amplificador, los cuales sintetizan la señal considerando las limitaciones de la tarjeta Arduino. El diseño propuesto presenta una plataforma electrónica que permitirá el entendimiento de los sistemas de radio basados en software.

Software Radio, Octave, Arduino

\begin{abstract}
Nowadays, the development of software radio based systems has allowed the transmission of data at high speeds and frequencies in the order of Gigahertz (Jovanovic, 2018). For this, the digital system is characterized by using digital modulation techniques in software through transmission symbols in the DigitalEnd stage, applying digital-analog (DAC) and analogdigital (ADC) converters to vary the sampling frequency and transmit the data in the Front End, (Chien, 2001). This work presents a digital educational platform to simulate the characteristics of a Software Radio system and not a web platform. The present investigation is focused on the characterization of signals modulated by Octave considering in the first instance the quadrature modulation QPSK, where the transmission of the modulated signal is carried out by means of the Arduino board in a digital port, with a digital-analog approach (DAC) using PWM modulation a RC filter and an amplifier circuit, which synthesize the signal considering the limitations of the Arduino board. The proposed design presents an electronic platform that will allow the understanding of software-based radio systems.
\end{abstract}

Citación: SILVA-CRUZ, Eric Mario, CABALLERO-JULIÁN, Franco Gabriel, PÉREZ-SOLANO, Miguel Ángel y PÉREZ-OJEDA, Cristian. Plataforma educativa para desarrollo de sistemas de software radio mediante modulación QPSK en Octave y Arduino. Revista de Educación Técnica. 2020. 4-12:22-31.

\footnotetext{
* Correspondencia del Autor (Correo Electrónico: eric.cruz@itoaxaca.edu.mx)

$\uparrow$ Investigador contribuyendo como primer autor.
} 


\section{Introducción}

Los sistemas de radio comunicación definidos por Software (SDR) o Software Radio, son implementados con sistemas digitales basadas en microprocesadores o sistemas embebidos, los cuales permiten realizar modulaciones digitales y el tratamiento de la señal aplicando técnicas de procesamiento digital de señales, está característica permitió sustituir los sistemas analógicos tradicionales, (Chien, 2001).

Los sistemas de comunicaciones basados en Software Radio se pueden implementar en microcontroladores, microprocesadores o bien en sistemas reconfigurables como lo son los campos de arreglos de compuertas reprogramables (FPGA), mediante estos dispositivos es posible implementarse algoritmos a frecuencias de reloj en el orden de Mega-hertz, (Biyoghe, 2017).

Los requerimientos para el diseño de este tipo de plataformas de hardware requieren una inversión alta para realizar todos los procesos que realiza un sistema de comunicación, por lo cual, se requieren de sistemas que operan a altas frecuencias y que además sea posible sintetizar la señal modulada. Así mismo, aplicar técnicas digitales para variar la tasa de muestreo en la adecuación y tratamiento digital de la señal (Harris, 2004), lo cual es definido en el sistema de comunicación digital como Digital-End, (Harada, 2002). El proceso para enviar la señal del Digital-End al canal de comunicaciones requiere de la conversión Digital Analógica, así como el uso de dispositivos analógicos en la etapa del sistema que es conocida como Front-End, tal como se muestra en la figura 1.

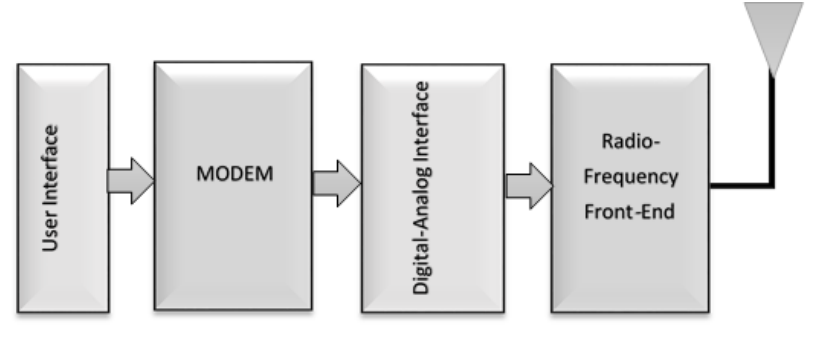

Figura 1 Digital-End y Front-End Fuente: Chien C. (2001)

\section{Planteamiento del problema}

En la actualidad existen una gran variedad de dispositivos para realizar la transmisión de señales, los cuales pueden sintetizar sistemas de comunicaciones básicos, permitiendo realizar procesos de obtención de señales, procesamiento y acondicionamiento de la señal, (Proakis, 2013).

Existen en el mercado sistemas electrónicos que permiten generar y analizar técnicas de modulación analógicas, como son la modulación AM y FM, sin embargo, no existen elementos educativos para generar sistemas de modulación digitales, en los cuales sea posible simular un sistema digital, siendo únicamente posible mediante el empleo de sistemas como los FPGA.

Los elementos básicos que requiere un sistema de Software Radio son; una fuente analógica de señal de datos acondicionada de forma digital con un $\mathrm{ADC}$, un procesador que module los datos mediante técnicas digitales, en donde, la señal de información se adecua en constelaciones de grupos de bits, como es el caso de la modulación QPSK, estos elementos en conjunto definen la señal en el Digital - End del transmisor.

Una vez realizado el tratamiento de los datos, estos se acondicionan para ser enviados a través de un canal de comunicaciones, por medio de aplicar técnicas de conversión Digital-Analógico (DAC) y haciendo uso de la etapa de Front-End, la señal se adecua al medio mediante mezcladores de señal, amplificadores, acopladores de línea de transmisión o antena según sea el caso del canal de comunicación alámbrico o inalámbrico.

Posteriormente la señal emitida pasa a través del canal de comunicaciones y se recibe en el receptor, cuya función es adquirir la señal y digitalizarla a partir de una conversión analógica a digital (ADC). Con el propósito de recibir correctamente la señal emitida el muestreo se debe realizar correctamente cumpliendo el criterio de Nyquist-Shannon, para posteriormente demodular los datos, y de ser necesario dar tratamiento a la señal para obtener la señal de información originada en la fuente. 


\section{Propuesta de solución}

En el presente artículo se propone el desarrollo de una plataforma que permita implementar sistemas de Software Radio básicos, mediante el cual pueda implementarse los procesos de acondicionamiento, procesamiento, transmisión y recepción de las señales digitales, las cuales son realizados en plataformas de desarrollo más complejas y costosas.

Para este propósito se parte de la idea de la implementación de un sistema de comunicaciones QPSK definido en (Pérez, 2017), en donde se considera la transmisión digital de una señal QPSK mediante una simulación de datos en la tarjeta Arduino Uno y la generación de señales en MATLAB, sin embargo, el proceso de diseño considera al sistema Arduino como elemento de procesamiento de la señal.

El sistema de software radio que aquí se propone consiste en definir el Digital End del transmisor y receptor a la programación mediante el software Octave y considerar a la tarjeta Arduino como un elemento de acondicionamiento de señal en el transmisor y receptor simulando el Front-End del sistema Software Radio, y que mediante esta configuración se considere una plataforma reconfigurable, la cual es la característica principal de los sistemas basados en Software Radio.

En la sección de metodología a desarrollar se explica brevemente las partes que integran la plataforma de Software Radio basada en Octave y Arduino. Para el diseño de la plataforma, en la sección de Desarrollo se definen los conceptos y los elementos utilizados para caracterizar e implementar el sistema.

\section{Metodología}

La metodología de diseño para realizar la plataforma de Software Radio basada en Octave y Arduino se caracteriza por los siguientes elementos:

- Los datos binarios se modulan digitalmente en QPSK mediante la agrupación de pares de bits para generar los símbolos a transmitir en Octave.
- Los valores de la señal analógica a transmitir se acondicionan mediante el escalamiento de valores negativos a positivos únicamente, esto debido a la característica de la señal a transmitir.

- Se modula por ancho de pulso (PWM) la señal para ser transmitida a través de un puerto digital de Arduino y así pueda ser emitida al canal de comunicación.

- Se modela un circuito que permita aproximar a una conversión Digitalanalógica usando una configuración Resistiva Capacitiva (RC) y un Amplificador Operacional.

- La señal será recibida en un puerto analógico del Arduino, en donde se realizará la conversión analógica a digital mediante la resolución del ADC interno.

- Los datos obtenidos por el firmware instalado en Arduino se enviarán al puerto serial a Octave para comparar la señal transmitida y recibida.

\section{Desarrollo}

a) Octave

El entorno de programación Octave está basado en el sistema de programación MATLAB, siendo esta su versión libre. Este software nos permite caracterizar algoritmos de programación mediante algebra lineal para el procesamiento digital de señales (DSP), al realizar operaciones con vectores y matrices para determinar el comportamiento de los datos y señales aplicadas en diversas áreas de la ingeniería, (Quarteroni, 2006).

En la plataforma de programación Octave se generar la señal QPSK mediante la cual se adecuan los datos binarios a señales sinusoidales a transmitir. En el receptor Octave obtiene la señal analógica a partir de la señal de Arduino y reconstruye la señal que se generó en el transmisor. La conectividad de Octave y Arduino se realiza de forma serial (Margolis, 2011), lo cual representa una ventaja para el envío y procesamiento de señales en el diseño de la plataforma. 
Las técnicas de procesamiento digital de señales son implementadas mediante algoritmos programables que se implementan en circuitos digitales combinacionales y secuenciales.

\section{b) Modulación QPSK}

La señal QPSK se define mediante la ecuación 1, en donde cada elemento a transmitir corresponde a un símbolo:

$S_{n}(t)=\sqrt{\frac{2 E_{s}}{T_{s}}} \sin \left(2 \pi f_{c} t+(2 n-1) \frac{\pi}{4}\right)$

Donde:

$s_{n}$ : Símbolo modulado, $\mathrm{n} \in\{1,2,3,4\}$.

$f_{c}$ : Frecuencia portadora.

$E_{S}$ : Energía del símbolo.

$T_{S}$ : Duración del símbolo.

Los datos digitales en grupos de bits son modulados mediante la representación de señal QPSK, la cual realiza una agrupación de pares de bits, figura 2, mismos que definen la señal en cuadratura con una constelación de 4 fases y se denotan por símbolos sinusoidales.

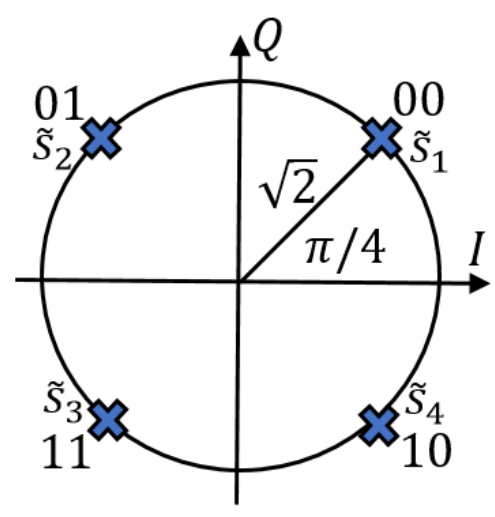

Figura 2 Constelación de Señal QPSK Fuente: Chien C., (2001)

El diseño la señal binaria se representa en forma de señal sinusoidal con cambios de fase y representa la señal modulada a transmitir, considerando un receptor coherente con respecto a la fase.

c) Modulación por ancho de pulso (PWM)
Una vez que se ha realizado la modulación en Octave el proceso siguiente es adecuar la señal digital al canal de comunicación, lo cual se logra mediante una modulación PWM, en donde se tiene una modulación por ancho de pulso que corresponde a una señal rectangular cuyo ciclo de trabajo varía y representa una aproximación a una señal sinusoidal en términos de potencia, (Goldberg, 1999).

En la metodología de diseño, la señal PWM únicamente emplea valores positivos para modular la señal, (Intel® Quark, 2015), la cual se asemeja a una Look-up Table (LUT). Por lo cual cada valor a transmitir en QPSK debe ajustarse a valores positivos y caracterizar la señal PWM, y así poder ser transmitida en el puerto digital de la tarjeta Arduino.

d) Transmisor Arduino.

Arduino UNO es una plataforma electrónica que permite realizar la adecuación de la señal al canal de comunicación alámbrico, mediante el uso de sus puertos analógicos y digitales, plataforma que emplea como base un sistema microcontrolado ATMega.

La versión UNO de Arduino únicamente posee puertos de entrada Analógicos en donde se realiza una conversión Analógica a Digital con una resolución de 10 bits, los cuales permiten una codificación de1024 datos en forma digital, que se adecua a los propósitos de diseño de la plataforma.

En lo que corresponde a los puertos digitales, Arduino no ofrece las características de conversión Analógica a Digital, sin embargo, posee la modulación PWM con una resolución de 8 bits a diferentes intervalos de frecuencia y para el diseño de la plataforma propuesta nos permite sintetizar los datos a señales analógicas, dicha operación de sintetizado puede realizarse con otros microcontroladores (Intel® Quark, 2015), esto al conectar en serie la señal de salida del puerto a un circuito RC y un amplificador Operacional. 


\section{e) Circuito de Filtrado}

El diseño mediante una modulación PWM y un circuito de filtro RC nos permite hacer una aproximación a la señal obtenida mediante un conversor Digital Analógico, existiendo una amplia variedad de circuitos y configuraciones que realizan esta operación, entre los cuales encontramos los arreglos:

- $\quad$ Circuito PWM y filtro RC.

- Circuito PWM y filtro RC con amplificador operacional.

Al ser filtrada la señal con el arreglo correspondiente, se obtiene una señal Analógica adecuada en términos de voltaje, y será la señal que recibirá el Arduino Receptor, con un voltaje de 0 a $5 \mathrm{~V}$ en uno de sus puertos analógico del, tal como se observa en la figura 3.

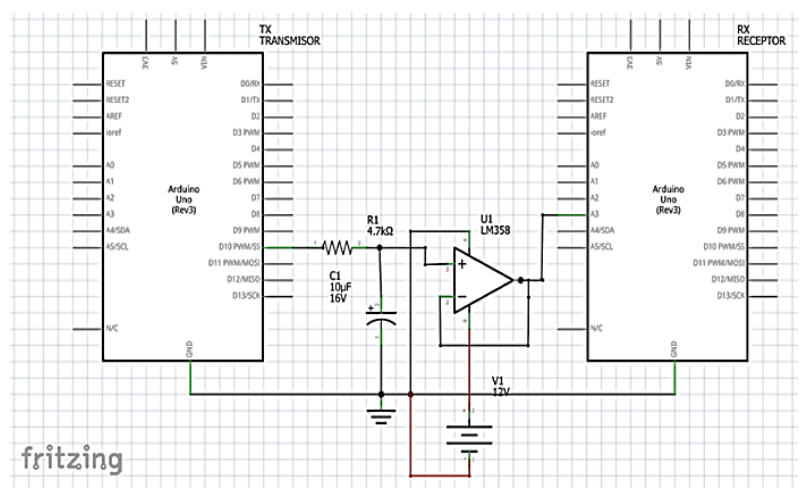

Figura 3 Transmisor y Receptor Front-End con Arduino. generado en Fritzing

Fuente: Elaboración propia

\section{f) Receptor Arduino}

El Arduino receptor adquiere la señal en uno de sus puertos digitales y la adecua mediante su conversor Analógico digital, considerando que la señal PWM son los valores codificados y que pueden ser leído e interpretados mediante un firmware.

\section{g) Firmware en Arduino}

Los procesos de configurar, transmitir, adecuar e interpretar la señal que se transmite y envía son llevados a cabo mediante un software instalado en la tarjeta Arduino y programando mediante la interfaz del entorno de desarrollo (IDE) de Arduino, se denomina firmware debido a que el programa al instalarse no se puede modificar cuando está en ejecución, sin embargo, es reprogramable.
A continuación, se presenta el código firmware para el Arduino Transmisor:

int $\mathrm{pwmOut}=10$.

float val $=0$.

float valr $=0$.

Byte.

valx 504[]$=\{217,192,163,131,99,69,43$, $2,7,0,1,10,26,49,76,107,139,170,199,222,240$, $51,254,249,237,217,192,163,131,99,69,43,22,7$, $0,1,10,26,49,76,107,139,170,199,222,240,251,2$ $54,249,237,217,192,163,131,99,69,43,22,7,0,1$, $10,26,49,76,107,139,170,199,222,240,251,254$, $249,237,217,192,163,131,99,69,43,22,7,0,1,10$, $26,49,76,107,139,170,199,222,240,251,254,249$ ,237,217,217,237,249,254,251,240,222,199,170 ,139,107,76,49,26,10,1,0,7,22,43,69,99,131,163 ,192,217,237,249,254,251,240,222,199,170,139 ,107,76,49,26,10,1,0,7,22,43,69,99,131,163,192 ,217,237,249,254,251,240,222,199,170,139,107 ,76,49,26,10,1,0,7,22,43,69,99,131,163,192,217 ,237,249,254,251,240,222,199,170,139,107,76, $49,26,10,1,0,7,22,43,69,99,131,163,192,217,37$, $17,5,0,3,14,32,55,84,115,147,178,205,228,244$, $253,254,247,232,211,185,155,123,91,62,37,17$, $5,0,3,14,32,55,84,115,147,178,205,228,244,253$ ,254,247,232,211,185,155,123,91,62,37,17,5,0, $3,14,32,55,84,115,147,178,205,228,244,253,25$ $4,247,232,211,185,155,123,91,62,37,17,5,0,3,1$ $4,32,55,84,115,147,178,205,228,244,253,254,2$ $47,232,211,185,155,123,91,62,37,37,62,91,123$, $155,185,211,232,247,254,253,244,228,205,178$, $147,115,84,55,32,14,3,0,5,17,37,62,91,123,155$, $185,211,232,247,254,253,244,228,205,178,147$, $115,84,55,32,14,3,0,5,17,37,62,91,123,155,185$, $211,232,247,254,253,244,228,205,178,147,115$, $84,55,32,14,3,0,5,17,37,62,91,123,155,185,211$, $232,247,254,253,244,228,205,178,147,115,84,5$ $5,32,14,3,0,5,17,37,217,237,249,254,251,240,2$ $22,199,170,139,107,76,49,26,10,1,0,7,22,43,69$, $99,131,163,192,217,237,249,254,251,240,222,1$ $99,170,139,107,76,49,26,10,1,0,7,22,43,69,99,1$ $31,163,192,217,237,249,254,251,240,222,199,1$ $70,139,107,76,49,26,10,1,0,7,22,43,69,99,131,1$ $63,192,217,237,249,254,251,240,222,199,170,1$ $39,107,76,49,26,10,1,0,7,22,43,69,99,131,163,1$ 92,217\}; // QPSK signal simulation

$$
\text { int delayt }=50 \text {; }
$$

void $\operatorname{setup}()$

SILVA-CRUZ, Eric Mario, CABALLERO-JULIÁN, Franco Gabriel, PÉREZ-SOLANO, Miguel Ángel y PÉREZ-OJEDA, Cristian. Plataforma educativa para desarrollo de sistemas de software radio mediante modulación QPSK en Octave y Arduino. Revista de Educación Técnica. 2020 
\{

pinMode(pwmOut, OUTPUT);

Serial.begin(9600);

\}

void loop ()

\{

for (byte $\mathrm{i}=0 ; \mathrm{i}<=504 ; \mathrm{i}++$ )

\{

analogWrite (pwmOut,valx504[i]);

delay(delayt);

Serial.println(valx504[i]);

\}

\}

Así mismo se presenta el código firmware para el Arduino Receptor:

float val $=0$;

float valr $=0$;

float $\operatorname{vrx}=0$

int

valx6[] $=\{0,0,0,0,0,0,0,0,0,0,0,0,0,0,0,0$, $0,0,0,0,0,0,0,0,0,0,0,0,0,0,0,0,0,0,0,0,0,0,0,0,0,0$ , $0,0,0,0,0,0,0,0,0,0,0,0,0,0,0,0,0,0,0,0,0,0,0\}$;

int delayt $=500$;

void setup ()

\{

Serial.begin(9600);

\}

void loop ()

\{

for (int $i=0 ; i<=64 ; i++)$
\{

vrx=analogRead(A3);

valx6[i] $=$ vrx $/ 4$;

Serial.println("indice i:");

Serial.println(i);

Serial.println("Valor en i:");

Serial.println(valx6[i]);

delay(delayt);

$$
\}
$$

\}

El código desarrollado considera la transmisión de señales binarias tal como se puede observar en el Gráfico 1, las cuales son moduladas en cuatro símbolos que determinan las fases QPSK.

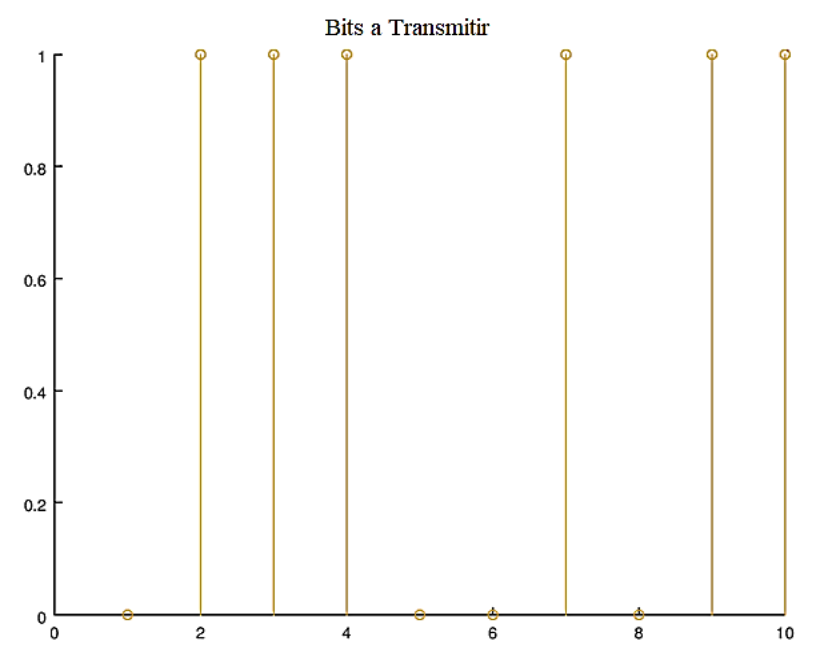

Gráfico 1 Señal Binaria generada en Octave Fuente: Elaboración propia

Las ecuaciones (2) a (5) corresponden a los distintos símbolos que representan las señales cuadráticas en la constelación QPSK y con cambios de fase generadas en Octave, ver Gráfico 2.

$$
\begin{aligned}
& \operatorname{sym} 1=A * \sin \left(w_{c} * 2 * t b+5 * \mathrm{pi} / 4\right) \\
& \operatorname{sym} 2=A * \sin \left(w_{c} * 2 * t b+3 * \mathrm{pi} / 4\right) ; \\
& \operatorname{sym} 3=A * \sin (w c * 2 * t b+7 * \mathrm{pi} / 4) ; \\
& \operatorname{sym} 4=A * \sin (w c * 2 * t b+\mathrm{pi} / 4) ;
\end{aligned}
$$


Tomando en consideración las ecuaciones de diseño de los símbolos, a continuación, se muestra el código de simulación de Octave para el sistema QPSK y los datos a transmitir.

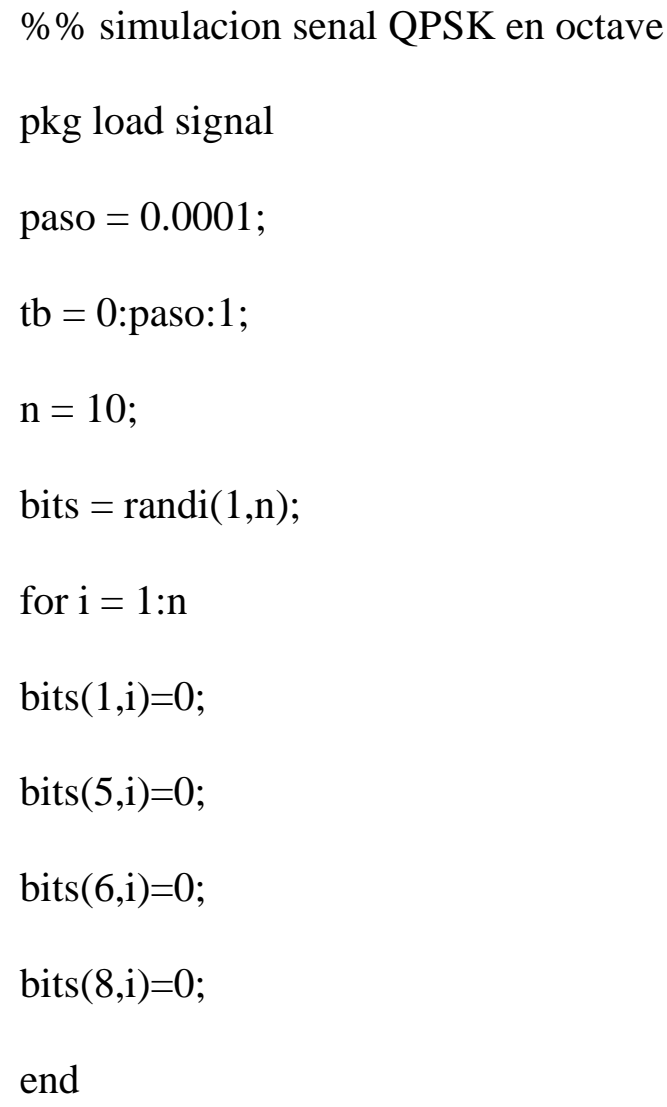

subplot(2,2,2); plot(sym2); title('01');

subplot(2,2,3); plot(sym3); title('10');

subplot(2,2,4); plot(sym4); title('11');

$\bmod =[]$

for $i=1: 2: n-1$

if $(\operatorname{bits}(\mathrm{i})==0 \& \& \operatorname{bits}(\mathrm{i}+1)==0) \bmod =$ [mod sym1];

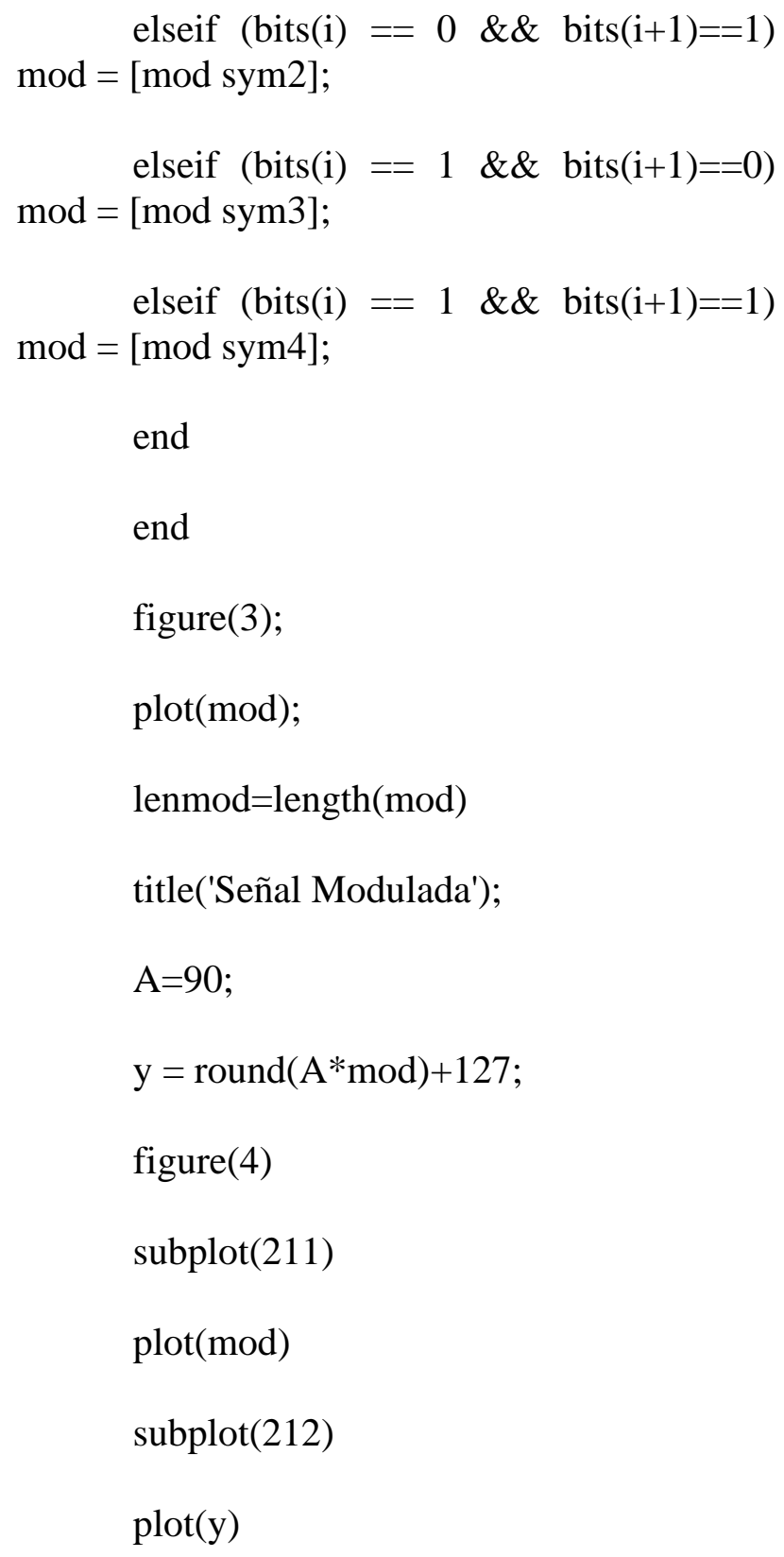

SILVA-CRUZ, Eric Mario, CABALLERO-JULIÁN, Franco Gabriel, PÉREZ-SOLANO, Miguel Ángel y PÉREZ-OJEDA, Cristian. Plataforma educativa para desarrollo de sistemas de software radio mediante modulación QPSK en Octave y Arduino. Revista de Educación Técnica. 202 


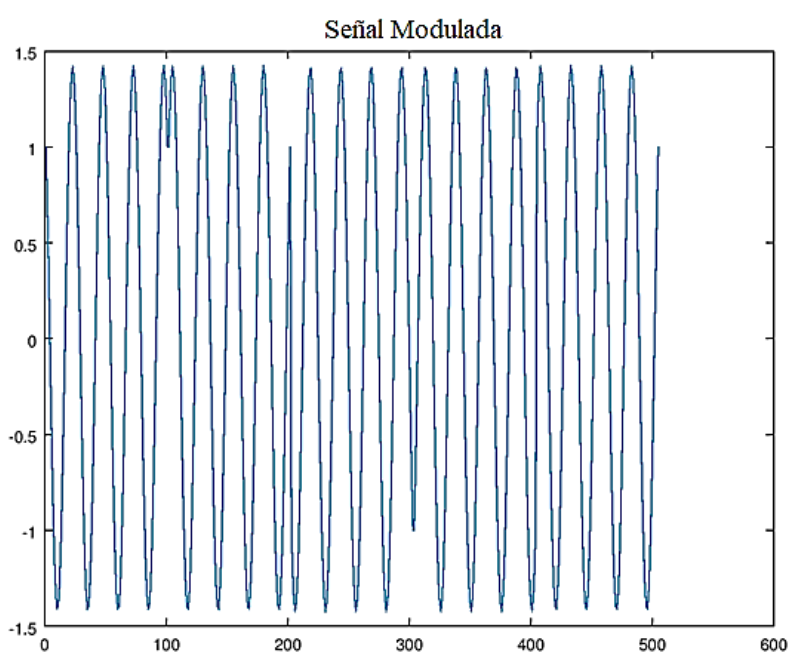

Gráfico 2 Señal QPSK transmitida en Octave Fuente: Elaboración propia

Una vez obtenida la señal modulada QPSK se realiza la adecuación al puerto digital mediante la modulación PWM. La señal PWM generada es una codificación de la señal sinusoidal con cambios de fase que depende de un contador para obtener la conversión a la frecuencia mínima de la señal de información, ver Gráfico 3.
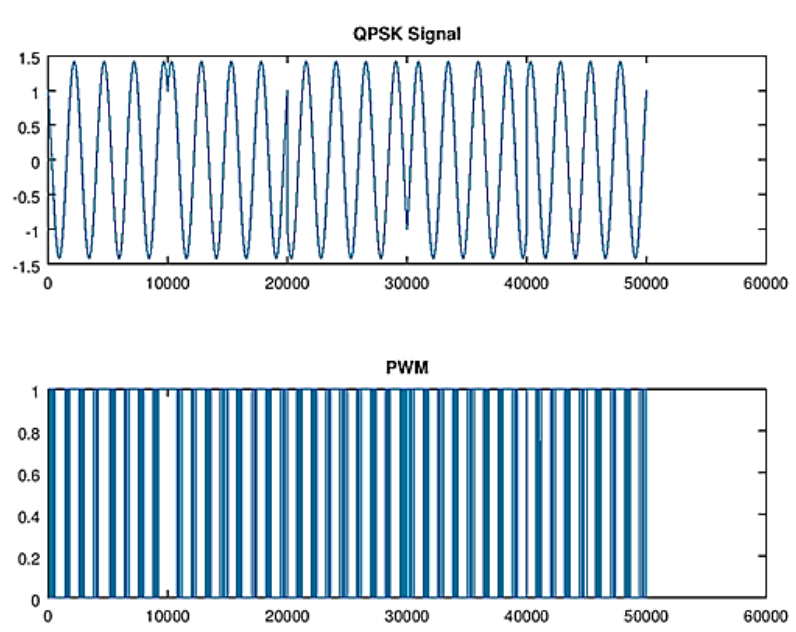

Gráfico 3 Señal QPSK sintetizada con PWM en Arduino Fuente: Elaboración propia

La señal modulada PWM se transmite en el puerto digital de Arduino, en este caso se eligió el pin digital 10. Este puerto conecta al circuito RC y amplificador que realiza la conversión a una señal analógica DAC, debido a los valores que representa la señal PWM, considerando un valor de conversión de $5 \mathrm{~V}$, el cual representa un valor aceptable en la entrada analógica del sistema Arduino en su puerto A3. Para obtener los datos de Octave a Arduino se realiza un acceso al puerto serial mediante un emulador, en este caso el software Virtual Serial Port Emulator, ver Gráfico 4.

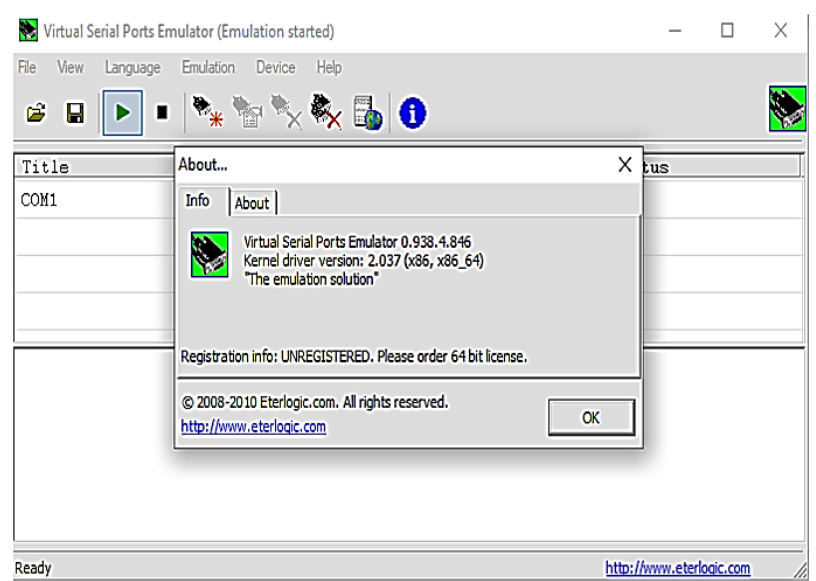

Gráfico 4 Virtual Serial Port Emulator Fuente: Elaboración propia

La señal obtenida con el código en Octave para acceder al puerto serial se observa en el Gráfico 5. En donde la simulación en Octave nos permite obtener la señal enviada mediante el siguiente código:

clc;clear all;close all;

pkg load instrument-control

pkg load signal

$\mathrm{s} 1=\operatorname{serial}("|| I|| \mid. C O M 1 ") \quad \%$ Open the virtual port

srl_flush(s1)

y_temp $=\operatorname{cell}(10,1)$

$\mathrm{y}=0$

while true

for $\mathrm{i}=1: 10$

y_serial

str2num(char(srl_read(s1,10)))

y_temp $\{\mathrm{i}, 1\}=$ y_serial

endfor

$\mathrm{y}=\operatorname{cat}\left(1, \mathrm{y}, \mathrm{y} \_\right.$temp $\left.\{1: 10\}\right)$

$\operatorname{plot}(\mathrm{y})$

\%pause(1) \% realiza una medición continua de datos

endwhile 
srl_close(s1)

fclose(s1);
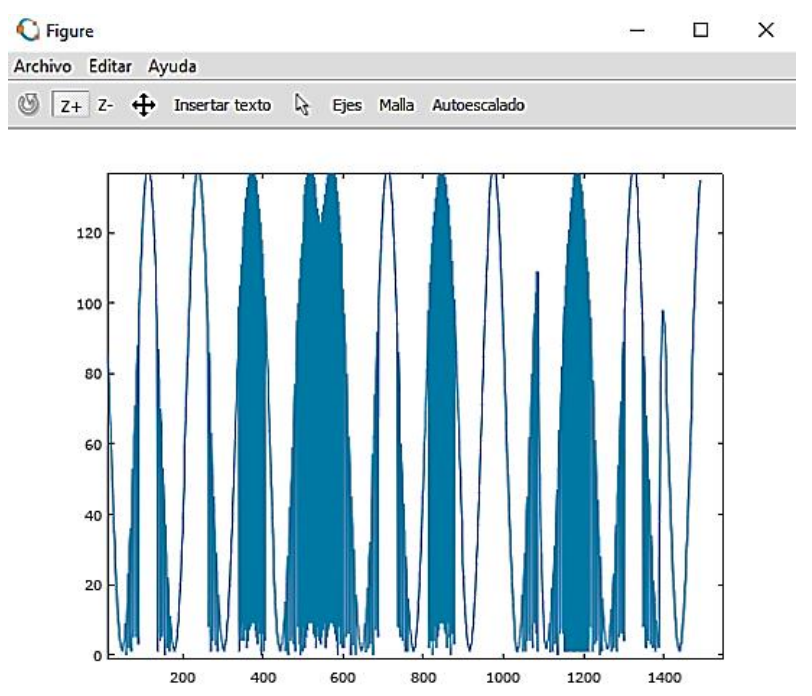

Gráfico 5 Señal QPSK recibida en Octave Fuente: Elaboración propia

Para el diseño del firmware del transmisor y receptor se deben tener ciertas consideraciones, entre la cuales se encuentra la velocidad de transmisión y recepción de datos, las cuales son requeridas para definir la frecuencia de generación de datos con PWM. Así mismo en el receptor debemos considerar la resolución del puerto que realiza la conversión ADC y muestrear correctamente la señal, esto considerando que la reconstrucción de la señal PWM la realizará el puerto analógico de Arduino Uno y se utilizará un receptor coherente, en donde la fase de la señal esta sincronizada.

\section{Conclusiones}

La presente investigación muestra el diseño de una plataforma educativa de Software Radio, en la cual se puede realizar simulaciones e implementaciones de sistemas de comunicación digitales a un bajo costo, con la capacidad de transmitir datos mediante sistemas de modulación utilizando en sistemas alámbricos e inalámbricos, como es el caso de la modulación QPSK.
Se considera una plataforma educativa debido a la integración de elementos que puedan ser aplicados en laboratorios de ingeniería y prácticas de comunicaciones digitales, emulando la transmisión y recepción de señales que caracterizan el sistema de Software Radio utilizando Octave y Arduino. En donde se abarcan los temas de conversión ADC y DAC, empleando la idea de sintetizar los datos mediante un conversor Digital Analógico con PWM, tal como lo realizan sistemas más elaborados con FPGA y microprocesadores.

El puerto analógico del Arduino receptor con respecto a la resolución nos permite realizar la obtención de los datos enviados y ser obtenidos en el software Octave, en donde es posible realizar un tratamiento a la señal, la cual modifica sus características al pasar por el canal de comunicación.

Esta plataforma nos permitió comprobar algoritmos de transmisión y recepción de datos, codificación de canal, así como modificar el canal de transmisión mediante el acondicionamiento del canal utilizando el software Octave, el cual representa una herramienta de desarrollo con un gran potencial al interactuar con la plataforma Arduino de forma serial.

Una de las limitaciones de esta plataforma es la velocidad de transmisión de los puertos y la lectura de los datos, así como la sincronización de la señal y estas son áreas de oportunidad para contribuir en el desarrollo de esta plataforma.

Así mismo se requiere el empleo de técnicas de procesamiento digital para tratar la señal de datos recibida, tal como observamos la señal contiene elementos no deseados debidos al canal de comunicaciones y al muestreo de la señal.

El desarrollo a futuro de la plataforma será realizar un sistema de filtrado digital para restringir la señal a los valores deseados, sincronizar y ecualizar la señal considerando canales de comunicaciones inalámbricos. 


\section{Referencias}

Biyoghe J. S. (2017). Design and implementation of a high data rate QPSK demodulator for Nanosatellites, (Tesis de Maestría), Faculty of Engineering at the Cape Peninsula University of Technology, Bellville Campus.

Chien C. (2001). Digital Radio Systems, Systems on a Chip, A Systems Approach. Rockwell Science Center. Kluwer Academic Publishers.

Goldberg, B. G. (1999). Digital frequency synthesis demystified, LHH Technology Publishing.

Harada H. \&\& Prasad R. (2002). Simulation and Software Rafio for Mobile Communications, Universal Personal Communications.

Harris, F. J. (2004). Multirate signal processing for communications systems. New Jersey, USA: Prentice Hall.

Intel ${ }^{\circledR}$ Quark (2015), Direct Digital Synthesis on the Intel ${ }^{\circledR}$ Quark $^{\mathrm{TM}}$ microcontroller D1000, White Paper. Recuperado de http://www.intel.com/design/literature.htm

Jovanovic, D. G. (Ed.). (2018). Advances in Multirate Systems, Mexico, Editorial Springer International Publishing.

Margolis M. (2011). Arduino Cookbook. O’Reilly Media, Inc.

Pérez A. L. (2017). Diseño de un transmisor y un receptor digital en Arduino, basados en la modulación de señales digitales. (Tesis de Grado de Licenciatura). Escuela Técnica Superior de Ingeniería, Universidad de Sevilla, Sevilla España.

Proakis J. G. \&\& Salehi M. \&\& Bauch G. (2013). Contemporary Communication Systems Using MATLAB®, Third Edition, Cengage Learning.

Quarteroni A. \&\& Saleri F. (2006). Cálculo Cientifico con MATLAB y Octave, SpringerVerlag. 